\title{
The Efficacy of EGFR-Tyrosine Kinase Inhibitor in Non-Small Cell Lung Cancer Patients With Synchronous Brain Metastasis: A Real-World Study
}

Jin-Hyuk Choi

Ajou University

Yong Won Choi

Ajou University

Hyun Woo Lee

Ajou University

Seok Yun Kang

Ajou University

Geum Sook Jeong

Ajou University

Mi Sun Ahn ( $\triangle$ maruhiran@hanmail.net)

Ajou University

Young-Taek Oh

Ajou University

O kyu Nho

Ajou University

Se-Hyuk Kim

Ajou University

Tae Hoon Roh

Ajou University

Seung Soo Sheen

Ajou University

\section{Research Article}

Keywords: EGFR, non-small cell lung cancer, brain metastasis

Posted Date: January 18th, 2021

DOI: https://doi.org/10.21203/rs.3.rs-137138/v1 
License: (c) (i) This work is licensed under a Creative Commons Attribution 4.0 International License. Read Full License

Version of Record: A version of this preprint was published at The Korean Journal of Internal Medicine on March 1st, 2022. See the published version at https://doi.org/10.3904/kjim.2021.315. 


\section{Abstract}

Background: The optimal treatment (Tx) for epidermal growth factor receptor (EGFR)-mutant non-small cell lung cancer (NSCLC) patients (pts) with brain metastasis (BM) remains to be determined.

Methods: A retrospective review was conducted on 77 NSCLC pts with synchronous BM who underwent first-line EGFR-TKI Tx (gefitinib: 46, erlotinib: 11, afatinib: 20). The outcomes of pts were analyzed according to the clinicopathological characteristics including local Tx modalities.

Results: Fifty-nine pts underwent local Tx for BM (gamma knife surgery (GKS): 37, whole brain radiotherapy (WBRT): 18, others: 4) concurrently or sequentially with EGFR-TKI. Pts treated with TKI alone showed significantly lower incidence of central nervous system (CNS) symptoms $(p=0.006)$, with a tendency toward a smaller number of BM lesions compared with those who received local treatment. The median progression-free survival (PFS) and overall survival (OS) after the initiation of EGFR-TKI for all pts were 9 and 19 months, respectively. In 60 pts with follow-up brain imaging, the median intracranial PFS was 15 months. Pts with EGFR exon 19 deletion $(n=42)$ had a significantly longer median OS than those with L858R mutation ( $n=25)$ or other mutations $(n=10)$ ( 23 vs. 17 months, $p=0.010)$. Other clinical characteristics, including CNS symptoms $(p=0.410)$, number of BM $(p=0.709)$, and the use of local Tx $(p=0.834)$, were not associated with OS, as well as PFS. In terms of the local optimal treatment modality, no difference was found between GKS and WBRT in the OS and PFS.

Conclusions: This study suggests that EGFR-TKI may result in a favorable outcome in NSCLC pts with synchronous BM, especially in deletion 19 mutant, regardless of the extent of BM lesions or local TX modalities. Pts with asymptomatic BM can be treated with EGFR-TKI and careful surveillance.

\section{Background}

Approximately $20-40 \%$ of non-small cell lung cancer (NSCLC) patients will develop brain metastasis (BM) during the course of their disease [1, 2]. In several studies especially from Asia, the frequency of synchronous or metachronous BM in epidermal growth factor receptor (EGFR)-mutant NSCLC patients (39-70\%) has been higher than that in patients with the EGFR-wild type (28-38\%) [3-5]. Before the development of targeted therapy, the median overall survival (OS) of an unselected population of NSCLC patients with BM including EGFR-mutant was generally poor, ranging from 3 to 15 months [1, 2].

Cytotoxic chemotherapeutics are largely ineffective intracranially because of their inability to cross the brain-blood-barrier (BBB). Therefore, the standard treatment for BM has traditionally been local intervention such as surgical resection, whole brain radiotherapy (WBRT), and stereotactic radiosurgery (SRS), with or without additional chemotherapy $[1,2,6$, 7]. Since the introduction of EGFR tyrosine kinase inhibitor (EGFR-TKI), several studies have shown that EGFR-mutant NSCLC patients with BM could achieve favorable outcomes when treated with EGFR-TKIs [7, 8]. However, the optimal treatment strategy, such as upfront EGFR-TKI or the addition of local treatment, remains to be determined. 
Therefore, we performed a retrospective analysis to identify the potential prognostic factors and determine the optimal incorporation of local treatment in EGFR-mutant NSCLC with synchronous BM.

\section{Methods}

\section{Study population}

We retrospectively reviewed the clinical data of primary metastatic or recurrent EGFR-mutant NSCLC patients with synchronous BM who had started EGFR-TKI as the first-line therapy at Ajou University Hospital, between July 2011 and June 2018. All patients had BM at the time of initial diagnosis or recurrence. Patients who had started first-line EGFR-TKI at other hospitals during this period were excluded. The patients received a daily EGFR-TKI treatment of $250 \mathrm{mg}$ of gefitinib or $150 \mathrm{mg}$ of erlotinib or $40 \mathrm{mg}$ of afatinib according to the discretion of the medical oncologists. In most patients, the treatment modalities (gamma-knife surgery (GKS) or surgical resection or WBRT) for BM were selected according to the decision of the multidisciplinary team, including medical oncologists, neurosurgeons, and radiation oncologists, based on the size and number of BM, age, Eastern Cooperative Oncology Group (ECOG) performance status (PS), and other individual factors. GKS was performed using a model C Leksell Gamma Knife and Leksell GammaPlan ${ }^{\circledR}$ version 10.1.1 planning system (Elekta Instruments $A B$, Stockholm, Sweden). The responses to EGFR-TKI were evaluated every 2-3 months with chest CT while the patients were on therapy. Brain MRI was performed at three months after GKS then repeated every six months in patients who underwent GKS, whereas it was performed according to the discretion of physicians in patients treated with WBRT or EGFR-TKI alone.

All procedures performed in the study involving human participants were carried out in accordance with the ethical standards of the institutional and/or national research committee and with the $1964 \mathrm{Helsinki}$ Declaration and its later amendments or comparable ethical standards. The protocol was reviewed and approved by the Institutional Review Board (IRB) of Ajou University Hospital (IRB approval no. AJIRBMED-MDB-19-394). The IRB decided to waive the informed consent for this study because it was a retrospective study using anonymized data. Some patients (19) in the present study cohort was included in a previous study [9] about the prognostic significance of EGFR mutation subtypes in metastatic NSCLC, not limited to BM. However, the follow-up of the patients was extended in the current study.

\section{Clinical review}

Data on the patients were collected, including patient characteristics (gender, age, ECOG PS, histology, disease status at diagnosis, metastasis site, number of BM, central nervous system (CNS) symptoms, type of EGFR mutation, type of EGFR-TKI, and treatment modality of BM) and survival information.

\section{Statistical analysis}

OS, progression-free survival (PFS), and intracranial (IC)-PFS were calculated using the Kaplan-Meier method. OS was defined as the time from the starting day of EGFR-TKI to death. Data on the survivors were censored at the last follow-up. PFS was defined as the time from the starting day of EGFR-TKI to 
disease progression or the death of any cause. IC-PFS was defined as the time from the start day of EGFR-TKI to IC progression. Data of the patients alive or who died without evidence of IC progression were censored at the last follow-up time or the date of death, respectively. The log-rank test was used to analyze the differences between the survival curves. The comparison of the categorical variables between groups was performed by Fisher's exact test. All statistical analyses were performed two-sided using SPSS version 23.0 for Windows.

\section{Results}

\section{Patient characteristics}

A total of 77 EGFR-mutant NSCLC with synchronous BM patients received first-line EGFR-TKI treatment at our institution. Table 1 summarizes the patients' clinicopathological characteristics. Among the 77 patients, $43(56 \%)$ were female, with a median age of 63 years $(23-84), 57(74 \%)$ were in ECOG PS 0 or 1 , and 44 (57\%) were never smokers. The pathologic subtype was adenocarcinoma in 73 (not specified in 2 patients, adenosquamous carcinoma and large cell carcinoma in 2 patients). All patients had primary metastatic disease except one patient with recurrence, and $52(68 \%)$ patients had extracranial and extrathoracic metastasis at the time of diagnosis. While $16(21 \%)$ and $20(26 \%)$ patients had single and > $10 \mathrm{BMs}$, respectively, CNS symptoms were observed in 30 patients (39\%). Exon 19 deletion was the most common subtype of EGFR mutation (42 patients, 55\%). The patients were treated with gefitinib (46 patients), erlotinib (11 patients), or afatinib (20 patients). 
Table 1

Patients characteristics

\begin{tabular}{|c|c|c|c|c|}
\hline Characteristics & $\begin{array}{l}\text { Total } \\
N(\%)\end{array}$ & $\begin{array}{l}\text { Local Tx } \\
N(\%)\end{array}$ & $\begin{array}{l}\text { TKI alone } \\
N(\%)\end{array}$ & $P$ value \\
\hline Gender & $43(56)$ & $33(56)$ & $10(56)$ & 1.000 \\
\hline Female & $34(44)$ & $26(44)$ & $8(44)$ & \\
\hline \multicolumn{5}{|l|}{ Male } \\
\hline Age (years) & $54(70)$ & $44(75)$ & $10(56)$ & 0.147 \\
\hline$<70$ & $23(30)$ & $15(25)$ & $8(44)$ & \\
\hline \multicolumn{5}{|l|}{$\geq 70$} \\
\hline Smoking & $44(57)$ & $35(59)$ & $9(50)$ & 0.589 \\
\hline No & $33(43)$ & $24(41)$ & $9(50)$ & \\
\hline \multicolumn{5}{|l|}{ Yes } \\
\hline PS $(E C O G)^{a}$ & $57(74)$ & $43(73)$ & $14(78)$ & 0.768 \\
\hline 0,1 & $20(26)$ & $16(27)$ & $4(22)$ & \\
\hline \multicolumn{5}{|l|}{$\geq 2$} \\
\hline EGFR mutation & $42(55)$ & $31(53)$ & $11(61)$ & 0.691 \\
\hline Del 19 & $25(32)$ & $19(32)$ & $6(33)$ & \\
\hline L858R & $10(13)$ & $9(15)$ & $1(6)$ & \\
\hline \multicolumn{5}{|l|}{ Others } \\
\hline Extracranial, extrathoracic metastasis & $25(32)$ & $19(32)$ & $6(33)$ & 1.000 \\
\hline No & $52(68)$ & $40(68)$ & $12(67)$ & \\
\hline Yes & & & & \\
\hline
\end{tabular}

a PS 0: 5 patients, PS 3: 2 patients

Tx treatment, TKI tyrosine kinase inhibitor, N number, PS performance status, ECOG Eastern Cooperative Oncology Group, Del 19 exon 19 deletion, BM brain metastasis, GKS gamma knife surgery, WBRT whole brain radiation therapy, CNS central nervous system. 


\begin{tabular}{|c|c|c|c|c|}
\hline Characteristics & $\begin{array}{l}\text { Total } \\
N(\%)\end{array}$ & $\begin{array}{l}\text { Local Tx } \\
N(\%)\end{array}$ & $\begin{array}{l}\text { TKI alone } \\
N(\%)\end{array}$ & $P$ value \\
\hline Number of BM & $16(21)$ & $10(17)$ & $6(33)$ & 0.176 \\
\hline 1 & $14(18)$ & $9(15)$ & $5(28)$ & 0.051 \\
\hline $2-3$ & $27(35)$ & $23(39)$ & $4(22)$ & \\
\hline $4-10$ & $20(26)$ & $17(29)$ & $3(17)$ & \\
\hline$>10$ & & $19(32)$ & $11(61)$ & \\
\hline $1-3$ & & $40(68)$ & $7(39)$ & \\
\hline \multicolumn{5}{|l|}{$\geq 4$} \\
\hline Local Tx modality for BM & \multicolumn{4}{|l|}{$37(48)$} \\
\hline GKS & \multicolumn{4}{|l|}{$18(23)$} \\
\hline WBRT & \multicolumn{4}{|l|}{$2(3)$} \\
\hline Surgical resection + GKS & \multicolumn{4}{|l|}{$2(3)$} \\
\hline Surgical resection + WBRT & \multicolumn{4}{|l|}{$18(23)$} \\
\hline \multicolumn{5}{|l|}{ Not done } \\
\hline CNS symptoms & $47(61)$ & $31(52)$ & $16(89)$ & \multirow[t]{3}{*}{0.006} \\
\hline No & $30(39)$ & $28(48)$ & $2(11)$ & \\
\hline \multicolumn{4}{|l|}{ Yes } & \\
\hline TKI & $46(60)$ & $35(59)$ & $11(61)$ & \multirow[t]{4}{*}{1.000} \\
\hline Gefitinib & $11(14)$ & $9(15)$ & $2(11)$ & \\
\hline Erlotinib & $20(26)$ & $15(25)$ & $5(28)$ & \\
\hline \multicolumn{4}{|l|}{ Afatinib } & \\
\hline \multicolumn{5}{|c|}{ a PS 0: 5 patients, PS 3: 2 patients } \\
\hline \multicolumn{5}{|c|}{$\begin{array}{l}\text { Tx treatment, TKI tyrosine kinase inhibitor, N number, PS performance status, ECOG Eastern } \\
\text { Cooperative Oncology Group, Del } 19 \text { exon } 19 \text { deletion, BM brain metastasis, GKS gamma knife } \\
\text { surgery, WBRT whole brain radiation therapy, CNS central nervous system. }\end{array}$} \\
\hline
\end{tabular}

Eighteen patients (23\%) were treated with TKI alone and the remaining patients received additional local treatment (GKS: 37; WBRT: 18; GKS or WBRT after surgical resection: 4). The majority of patients with local treatment underwent or started the therapy before the initiation of TKI (41 patients), while 13 and 5 patients began the therapy after or on the same day, respectively. Patients treated with TKI alone showed a significantly lower incidence of CNS symptoms $(11 \%$ vs. $48 \%, p=0.006)$ with a tendency toward having 
a smaller number of BM lesions compared with those who received local treatment (Table 1). According to the local treatment modalities, the proportions of patients with poor PS and >10 BMs were higher in patients receiving WBRT than in those who underwent GKS (Table 2). 
Table 2

Patients characteristics according to local treatment modalities

\begin{tabular}{|c|c|c|c|}
\hline Characteristics & $\begin{array}{l}\text { GKS }^{a} \\
N(\%)\end{array}$ & $\begin{array}{l}\text { WBRT }^{\mathrm{b}} \\
N(\%)\end{array}$ & $P$ value \\
\hline $\begin{array}{l}\text { Gender } \\
\text { Female } \\
\text { Male }\end{array}$ & $\begin{array}{l}21(54) \\
18(46)\end{array}$ & $\begin{array}{l}12(60) \\
8(40)\end{array}$ & 0.784 \\
\hline $\begin{array}{l}\text { Age (years) } \\
<70 \\
\geq 70\end{array}$ & $\begin{array}{l}28(72) \\
11(28)\end{array}$ & $\begin{array}{l}16(80.0) \\
4(20.0)\end{array}$ & 0.547 \\
\hline $\begin{array}{l}\text { Smoking } \\
\text { No } \\
\text { Yes }\end{array}$ & $\begin{array}{l}22(56) \\
17(44)\end{array}$ & $\begin{array}{l}13(65) \\
7(35)\end{array}$ & 0.585 \\
\hline $\begin{array}{l}\text { PS (ECOG) } \\
0 / 1 \\
\geq 2\end{array}$ & $\begin{array}{l}32(82) \\
7(18)\end{array}$ & $\begin{array}{l}11(55) \\
9(45)\end{array}$ & 0.035 \\
\hline $\begin{array}{l}\text { EGFR mutation } \\
\text { Del } 19 \\
\text { L858R } \\
\text { Other }\end{array}$ & $\begin{array}{l}16(41) \\
15(39) \\
8(20)\end{array}$ & $\begin{array}{l}15(75) \\
4(20) \\
1(5)\end{array}$ & 0.054 \\
\hline $\begin{array}{l}\text { Extrathoracic metastasis } \\
\text { No } \\
\text { Yes }\end{array}$ & $\begin{array}{l}15(38) \\
24(62)\end{array}$ & $\begin{array}{l}4(20) \\
16(80)\end{array}$ & 0.239 \\
\hline Number of brain metastasi & & & \\
\hline$\leq 10$ & $35(90)$ & $7(35)$ & $<0.0001$ \\
\hline$>10$ & $4(10)$ & $13(65)$ & \\
\hline CNS symptoms & $22(56)$ & $9(45)$ & 0.425 \\
\hline $\begin{array}{l}\text { No } \\
\text { Yes }\end{array}$ & $17(44)$ & $11(55)$ & \\
\hline
\end{tabular}




\begin{tabular}{|llll|}
\hline Characteristics & GKS $^{\mathrm{a}}$ & WBRT $^{\mathrm{b}}$ & Pvalue \\
& $N(\%)$ & $N(\%)$ & \\
\hline
\end{tabular}

a,b including 2 patients with surgical resection, respectively

GKS gamma knife surgery, WBRT whole brain radiation therapy, Nnumber, PS performance status, ECOG Eastern Cooperative Oncology Group, Del 19 exon 19 deletion, CNS central nervous system.

\section{Treatment Outcome}

The median follow-up duration for the survivors was 32 months (22-67 months). One patient was excluded for outcome analysis because of lost to follow-up after seven days of TKI treatment. At the time of the last follow-up, 10 patients were still alive. The median PFS and OS of all patients after the initiation of TKI therapy were 9 and 19 months, respectively (Fig. 1). The median IC-PFS, analyzing 60 patients with available follow-up brain imaging, was 15 months (Fig. 1). The median OS of patients with exon 19 deletion was significantly longer than that of patients with other mutations ( $23 \mathrm{vs.} 17$ months, $p=0.010$ ). Other clinical characteristics, including the number of BM lesions, were not associated with the outcomes of patients (Table 3). The median OS, PFS and IC-PFS of patients who received local treatment were not different compared with those of the patients treated with TKI alone (22 vs. 19 months, $p=0.834 ; 9$ vs. 9 months, $p=0.182 ; 15$ vs. 19 months, $p=0.666$ ) (Fig. 2). 
Table 3

Progression-free survival and overall survival for all patients

\begin{tabular}{|c|c|c|c|c|}
\hline Prognostic factors & $\begin{array}{l}\text { Progression-free } \\
\text { survival } \\
\text { (months) }\end{array}$ & $\begin{array}{l}P \\
\text { value }\end{array}$ & $\begin{array}{l}\text { Overall } \\
\text { survival } \\
\text { (months) }\end{array}$ & $\begin{array}{l}P \\
\text { value }\end{array}$ \\
\hline Gender & 8 & 0.596 & 17 & 0.599 \\
\hline Female & 9 & & 23 & \\
\hline \multicolumn{5}{|l|}{ Male } \\
\hline Age (years) & 8 & 0.473 & 22 & 0.485 \\
\hline$<70$ & 10 & & 19 & \\
\hline \multicolumn{5}{|l|}{$\geq 70$} \\
\hline Smoking & 9 & 0.352 & 19 & 0.564 \\
\hline No & 8 & & 21 & \\
\hline \multicolumn{5}{|l|}{ Yes } \\
\hline PS (ECOG) & 9 & 0.231 & 23 & 0.246 \\
\hline 0,1 & 8 & & 11 & \\
\hline \multicolumn{5}{|l|}{$\geq 2$} \\
\hline EGFR mutation & 10 & 0.143 & 23 & 0.010 \\
\hline Del 19 & 8 & & 17 & \\
\hline \multicolumn{5}{|l|}{ Others } \\
\hline \multirow{2}{*}{$\begin{array}{l}\text { Extracranial, extrathoracic } \\
\text { metastasis }\end{array}$} & 9 & 0.333 & 24 & 0.441 \\
\hline & 8 & & 19 & \\
\hline No & & & & \\
\hline Yes & & & & \\
\hline
\end{tabular}

PS performance status, ECOG Eastern Cooperative Oncology Group, Del 19 exon 19 deletion, BM brain metastasis, Tx treatment, TKI tyrosine kinase inhibitor, CNS central nervous system. 


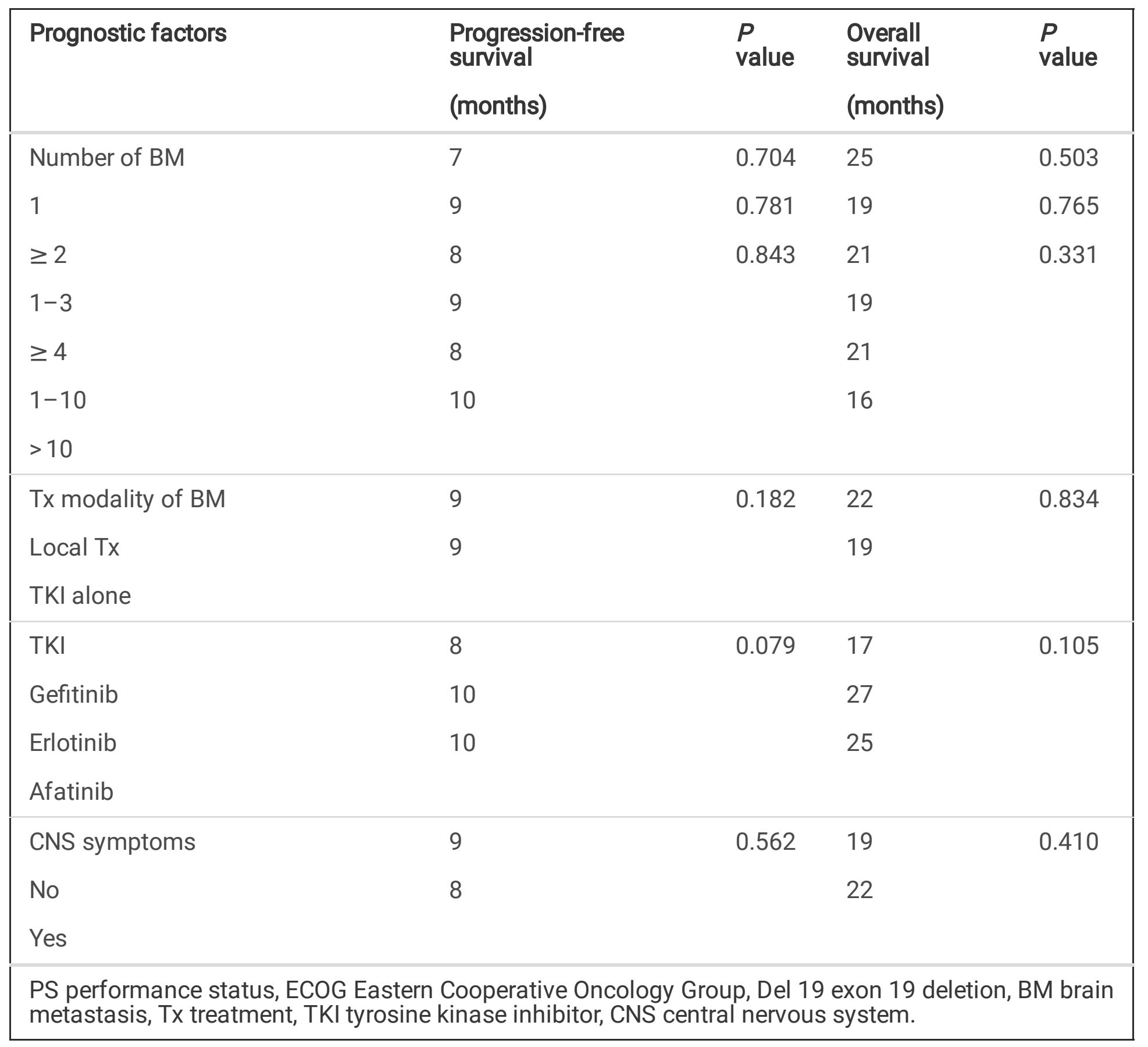

In the 47 patients without CNS symptoms (asymptomatic BM), 31 received local treatment, and 16 were treated with TKI alone. The median OS, PFS and IC-PFS were not different between the two groups (19 vs. 19 months, $p=0.443 ; 8$ vs. 10 months, $p=0.099 ; 15$ vs. 19 months, $p=0.692)$. In the 59 patients who received local treatment, no significant differences were observed between GKS and WBRT in OS (23 vs. 16 months, $p=0.296)$, PFS ( 9 vs. 8 months, $p=0.848$ ), and IC-PFS (13 vs. 26 months, $p=0.070$ ).

\section{Discussion}

The present study analyzed the outcomes of patients with EGFR-mutant NSCLC with BM who initiated first-line EGFR-TKI at our institution since 2011, when gefitinib had become reimbursable for first-line chemotherapy from the Korean national health insurance system. The outcomes of the current study 
cohort, including OS, PFS, and IC-PFS, were generally comparable with those of previous retrospective studies. In addition, we compared the treatment results of the present study with those of LUX-Lung 7, a randomized trial of afatinib versus gefitinib as a first-line treatment for EGFR-mutant NSCLC, with a preponderance of East Asian patients $[10,11]$. The median OS of the current study cohort was somewhat shorter than that of the LUX-Lung 7 trial (27.9/24.5 months) [11]. However, the LUX-Lung 7 trial included only ECOG PS 0 or 1 patients with a small proportion of patients with BM (16\%), whereas the present study consisted of a significant number of patients with ECOG PS 2 or $3(26 \%)$ [10]. Furthermore, the median OS of patients with ECOG 0 or 1 (23 months) in the present study was almost similar to that of the LUX-Lung 7 trial [11]. Moreover, the median PFS of the present study cohort (9 months) was comparable to that of patients with BM (7.2/7.4 months) in the LUX-Lung 7 trial [10]. These findings suggest that the current treatment outcomes of real-world practice are at least not inferior to those of clinical trial that consisted of selected patients with a relatively good health status, despite the limitation of an indirect comparison of two studies.

The current study demonstrated that exon 19 deletion was the only prognostic factor associated with favorable OS among various clinical characteristics. In patients with BM as well as unselected population of EGFR-mutant metastatic NSCLC including the cohort of our previous study, several studies showed better OS in patients with exon 19 deletion than in those with other mutations such as L858R $[1,9,12-$ 14].

The most controversial issue in the management of EGFR-mutant NSCLC with BM is the incorporation of local treatment. Although many retrospective studies showed conflicting results [5-8, 15-20], a few meta-analyses revealed better OS by addition of local treatment $[2,21,22]$. Furthermore, a large retrospective study from the United States demonstrated improved OS using upfront local treatment compared with EGFR-TKI alone [1]. The benefit of additional local treatment in NSCLC patients with BM treated with EGFR-TKI has the following biologic basis. First, many studies have shown that EGFR-mutant NSCLC is highly radiosensitive in both clinical and preclinical settings [1,23]. Second, radiation can disrupt blood-brain barrier, leading to increased penetration and cerebrospinal fluid concentration of EGFR-TKI [5, 20-22]. Third, EGFR-TKI may radiosensitize NSCLC cells [17, 22]. Finally, due to the discordance of EGFR mutations between the primary tumor and BM in some patients, EGFR-TKI can be ineffective to EGFR-wild type BM lesions [2, 5].

In terms of local modalities, SRS, such as GKS, combined with EGFR-TKI, seems to be the best approach because of its convenience and less toxicity, such as in neurocognitive dysfunction, and because several studies have indicated that it showed better OS than WBRT [1, 2, 21, 22, 24, 25]. However, in the present study, no difference was found in OS, PFS, and IC-PFS between GKS and WBRT despite the higher proportion of patients with poor PS and multiple BM lesions in the WBRT group. These results suggest that WBRT can be considered a useful alternative for patients unsuitable for SRS.

In contrast to previous large studies showing the advantage of additional local treatment $[1,2,17,20-$ 22], no significant difference was found in the OS, PFS, and IC-PFS between patients who underwent local 
treatment and those who were treated with EGFR-TKI alone in the present study. The significantly higher proportion of asymptomatic BM patients in the EGFR-TKI alone group and the absence of a significant difference in the outcomes between the local treatment and EGFR-TKI alone groups in patients without CNS symptoms may explain such results. Several retrospective studies have investigated the benefit of adding local therapy to EGFR-TKI in EGFR-mutant NSCLC patients with asymptomatic synchronous BM, but they showed conflicting results in terms of OS $[8,16,18,19]$. However, direct comparison with the current study seems to be difficult because some of these ones analyzed patient cohorts treated with WBRT alone [18] or including second or further line of EGFR-TKI therapy [8, 19]. Upfront EGFR-TKI may be a useful option for certain subgroups of EGFR-mutant NSCLC patients with BM such as those having no CNS symptom or a smaller number of BM lesions, given its antitumor activity in BM in addition to extracranial lesions $[5,7,20]$. Furthermore, the initiation of systemic treatment is not delayed with the avoidance of potential toxicity of local treatment [1]. However, a prospective randomized trial is essential to define the optimal treatment strategy for EGFR-mutant NSCLC patients with BM, although it seems to be difficult to conduct. In patients who receive upfront EGFR-TKI, close surveillance with brain imaging seems to be necessary to conduct a timely local treatment in case of IC progression.

This study analyzed all EGFR-mutant NSCLC patients with synchronous BM who had started EGFR-TKI as the first-line therapy in a single institution, during the defined period with a mature follow-up. Whereas many previous studies investigated WBRT alone cohorts or rather heterogenous population including second or more line of EGFR-TKI and metachronous BM [7, 8, 17-19], the current study analyzed only synchronous BM patients treated with first-line EGFR-TKI with more frequent usage of GKS than WBRT. Therefore, the results of the present study can reflect the treatment outcomes of real-world clinical practice in Korea.

However, this study has several limitations. First, this work is a retrospective analysis from a single institution with a relatively small sample size including patients treated with three types of EGFR-TKI. Second, the patients treated with local treatment only or best supportive care were not included in the analysis. Third, given the retrospective nature of the analysis, we did not analyze the potential toxicities associated with local treatment and their impact on quality of life. Fourth, because follow-up brain imaging was performed according to the discretion of the physician in many cases, not all patients were evaluated for IC-PFS. Finally, as osimertinib has been available in routine practice since December 2017 in Korea, the number of patients treated with third-generation EGFR-TKI such as osimertinib and olmutinib after progression was small (12 patients).

In conclusion, EGFR-TKI may result in favorable outcome in NSCLC patients with synchronous BM, especially in deletion 19 mutant, regardless of the extent of brain metastatic lesions or local treatment modalities. In addition, patients with asymptomatic BM can be treated with EGFR-TKI and careful surveillance.

\section{Abbreviations}


NSCLC

Non-small cell lung cancer; EGFR:Epidermal growth factor receptor; TKI:tyrosine kinase inhibitor; BM:Brain metastasis; PS:performance status; OS:overall survival; PFS:Progression-free survival; IC:intracranial; IRB:Institutional Review Board; ECOG; Eastern Cooperative Oncology Group; WBRT:Whole brain radiotherapy; SRS:Stereotactic radiosurgery; GKS:Gamma-knife surgery

\section{Declarations}

\section{Ethics approval and consent to participate}

The protocol was reviewed and approved by the Institutional Review Board (IRB) of Ajou University Hospital (IRB approval no. AJIRB-MED-MDB-19-394). The IRB decided to waive the informed consent for this study because it was a retrospective study using anonymized data.

\section{Consent for publication}

Not applicable

\section{Availability data and materials}

The datasets generated and/or analyzed during the current study are not publicly available due to the confidentiality of the data of patient but are available from the corresponding author on reasonable request.

\section{Competing interests}

The authors declare no competing interests.

\section{Funding}

This work was supported in part by Samyang Biopharmaceuticals Corporation, Korea. No grant number is applicable. The funder did not have any role in the design and conduct of study, the analysis and interpretation of the date, decision to publish and preparation of the manuscript.

\section{Authors' contributions}

JHC, YWC, SYK, GSJ, HWL, YTO, OKN, SHK, THR and MSA collected and analyzed clinical data. JHC and MSA wrote the main manuscript. JHC, MSA and SSS performed statistical analysis. All authors read and approved the final manuscript.

\section{Acknowledgments}

Not applicable.

\section{Authors' information}


${ }^{1}$ Department of Hematology-Oncology, Ajou University School of Medicine, Suwon, Korea, ${ }^{2}$ Department of Radiation Oncology, Ajou University School of Medicine, Suwon, Korea, ${ }^{3}$ Department of Neurosurgery, Ajou University School of Medicine, Suwon, Korea ${ }^{4}$ Department of Pulmonary and Critical Care Medicine, Ajou University School of Medicine, Suwon, Korea

\section{References}

1. Magnuson WJ, Lester-Coll NH, Wu AJ et al. Management of Brain Metastases in Tyrosine Kinase Inhibitor-Naive Epidermal Growth Factor Receptor-Mutant Non-Small-Cell Lung Cancer: A Retrospective Multi-Institutional Analysis. J Clin Oncol 2017; 35: 1070-1077.

2. Soon YY, Leong CN, Koh WY, Tham IW. EGFR tyrosine kinase inhibitors versus cranial radiation therapy for EGFR mutant non-small cell lung cancer with brain metastases: a systematic review and meta-analysis. Radiother Oncol 2015; 114: 167-172.

3. Ge M, Zhuang Y, Zhou X et al. High probability and frequency of EGFR mutations in non-small cell lung cancer with brain metastases. J Neurooncol 2017; 135: 413-418.

4. Hsu F, De Caluwe A, Anderson D et al. EGFR mutation status on brain metastases from non-small cell lung cancer. Lung Cancer 2016; 96: 101-107.

5. Saruwatari K, Ikeda T, Saeki S et al. Upfront Cranial Radiotherapy Followed by Erlotinib Positively Affects Clinical Outcomes of Epidermal Growth Factor Receptor-mutant Non-small Cell Lung Cancer With Brain Metastases. Anticancer Res 2019; 39: 923-931.

6. Chen $\mathrm{H}, \mathrm{Wu} \mathrm{A}, \mathrm{Tao} \mathrm{H}$ et al. Concurrent versus sequential whole brain radiotherapy and TKI in EGFRmutated NSCLC patients with brain metastasis: A single institution retrospective analysis. Medicine (Baltimore) 2018; 97: e13014.

7. Sung S, Lee SW, Kwak YK et al. Intracranial control and survival outcome of tyrosine kinase inhibitor (TKI) alone versus TKI plus radiotherapy for brain metastasis of epidermal growth factor receptormutant non-small cell lung cancer. J Neurooncol 2018; 139: 205-213.

8. Liu S, Qiu B, Chen L et al. Radiotherapy for asymptomatic brain metastasis in epidermal growth factor receptor mutant non-small cell lung cancer without prior tyrosine kinase inhibitors treatment: a retrospective clinical study. Radiat Oncol 2015; 10: 118.

9. Choi YW, Jeon SY, Jeong GS et al. EGFR Exon 19 Deletion is Associated With Favorable Overall Survival After First-line Gefitinib Therapy in Advanced Non-Small Cell Lung Cancer Patients. Am J Clin Oncol 2018; 41: 385-390.

10. Park K, Tan E-H, O'Byrne $\mathrm{K}$ et al. Afatinib versus gefitinib as first-line treatment of patients with EGFR mutation-positive non-small-cell lung cancer (LUX-Lung 7): a phase 2B, open-label, randomised controlled trial. Lancet Oncol 2016; 17: 577-589.

11. Paz-Ares $\mathrm{L}, \mathrm{Tan} E \mathrm{EH}, \mathrm{O}$ 'Byrne $\mathrm{K}$ et al. Afatinib versus gefitinib in patients with EGFR mutation-positive advanced non-small-cell lung cancer: overall survival data from the phase Ilb LUX-Lung 7 trial. Ann Oncol 2017; 28: 270-277. 
12. Jiang H, Zhu M, Li Y, Li Q. Association between EGFR exon 19 or exon 21 mutations and survival rates after first-line EGFR-TKI treatment in patients with non-small cell lung cancer. Mol Clin Oncol 2019; 11: 301-308.

13. Kim DW, Lee SH, Lee JS et al. A multicenter phase II study to evaluate the efficacy and safety of gefitinib as first-line treatment for Korean patients with advanced pulmonary adenocarcinoma harboring EGFR mutations. Lung Cancer 2011; 71: 65-69.

14. Iuchi T, Shingyoji M, Sakaida T et al. Phase II trial of gefitinib alone without radiation therapy for Japanese patients with brain metastases from EGFR-mutant lung adenocarcinoma. Lung Cancer 2013; 82: 282-287.

15. An N, Wang H, Li J et al. Therapeutic Effect Of First-Line EGFR-TKIs Combined With Concurrent Cranial Radiotherapy On NSCLC Patients With EGFR Activating Mutation And Brain Metastasis: A Retrospective Study. Onco Targets Ther 2019; 12: 8311-8318.

16. Chen Y, Wei J, Cai J, Liu A. Combination therapy of brain radiotherapy and EGFR-TKIs is more effective than TKIs alone for EGFR-mutant lung adenocarcinoma patients with asymptomatic brain metastasis. BMC Cancer 2019; 19: 793.

17. Chen Y, Yang J, Li X et al. First-line epidermal growth factor receptor (EGFR)-tyrosine kinase inhibitor alone or with whole-brain radiotherapy for brain metastases in patients with EGFR-mutated lung adenocarcinoma. Cancer Sci 2016; 107: 1800-1805.

18. Ke SB, Qiu H, Chen JM et al. Therapeutic Effect of First-line Epidermal Growth Factor Receptor Tyrosine Kinase Inhibitor (EGFR-TKI) Combined with Whole Brain Radiotherapy on Patients with EGFR Mutation-positive Lung Adenocarcinoma and Brain Metastases. Curr Med Sci 2018; 38: 10621068.

19. Wang W, Song Z, Zhang Y. Efficacy of brain radiotherapy plus EGFR-TKI for EGFR-mutated non-small cell lung cancer patients who develop brain metastasis. Arch Med Sci 2018; 14: 1298-1307.

20. Zhu Q Sun Y, Cui Y, Ye K, Yang C, Yang D, Ma J,Liu X, Yu J, Ge H. Clinical outcome of tyrosine kinase inhibitors alone or combined with radiotherapy for brain metastases from epidermal growth factor receptor (EGFR) mutant non small cell lung cancer (NSCLC). Oncotarget 2017; 8: 13304-13311.

21. Du XJ, Pan SM, Lai SZ et al. Upfront Cranial Radiotherapy vs. EGFR Tyrosine Kinase Inhibitors Alone for the Treatment of Brain Metastases From Non-small-cell Lung Cancer: A Meta-Analysis of 1465 Patients. Front Oncol 2018; 8: 603.

22. Wang C, Lu X, Lyu Z et al. Comparison of up-front radiotherapy and TKI with TKI alone for NSCLC with brain metastases and EGFR mutation: A meta-analysis. Lung Cancer 2018; 122: 94-99.

23. Johung KL, Yao X, Li F et al. A Clinical Model for Identifying Radiosensitive Tumor Genotypes in Non-Small Cell Lung Cancer. Clinical Cancer Research 2013; 19: 5523-5532.

24. Chang EL, Wefel JS, Hess KR et al. Neurocognition in patients with brain metastases treated with radiosurgery or radiosurgery plus whole-brain irradiation: a randomised controlled trial. Lancet Oncol 2009; 10: 1037-1044. 
25. Habets EJ, Dirven L, Wiggenraad RG et al. Neurocognitive functioning and health-related quality of life in patients treated with stereotactic radiotherapy for brain metastases: a prospective study. Neuro Oncol 2016; 18: 435-444.

\section{Figures}

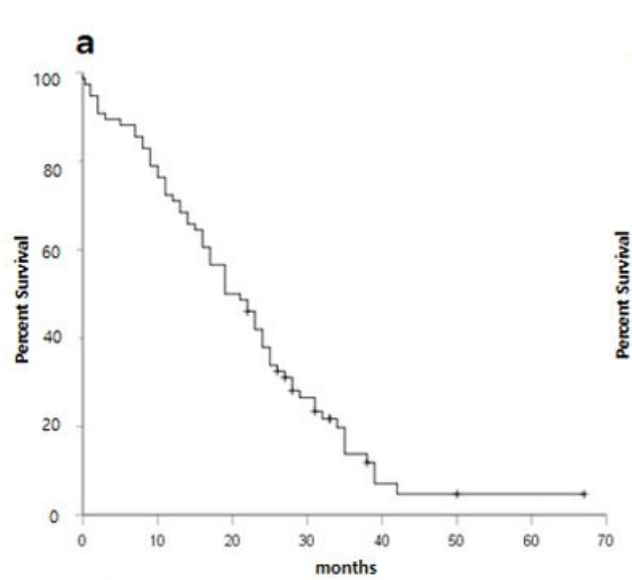

No. at risk

$76 \quad 58$

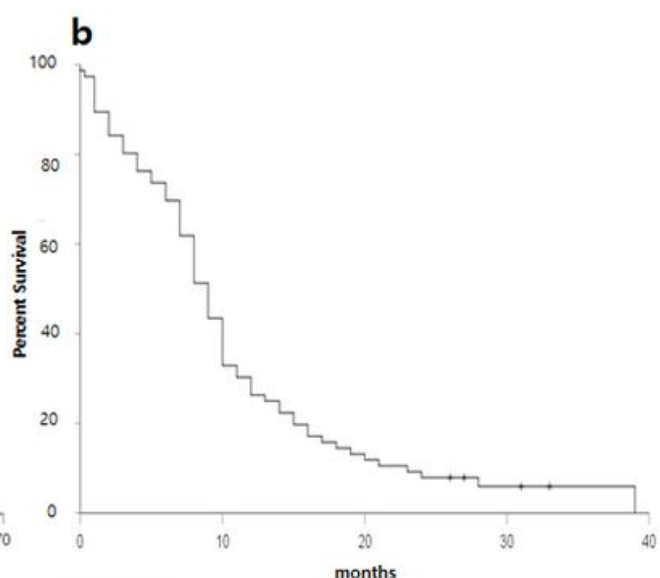

No. at risk

76

0

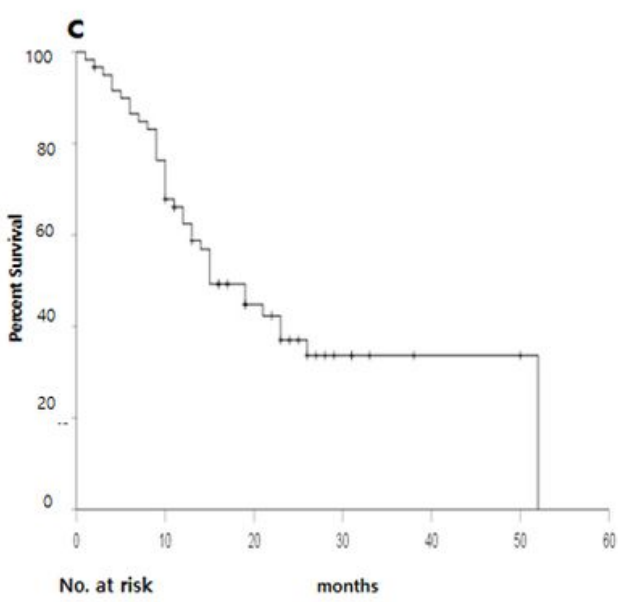

\section{Figure 1}

a Overall survival, b progression-free survival (PFS), and c intracranial-PFS of EGFR-mutant non-small cell lung cancer patients with brain metastasis
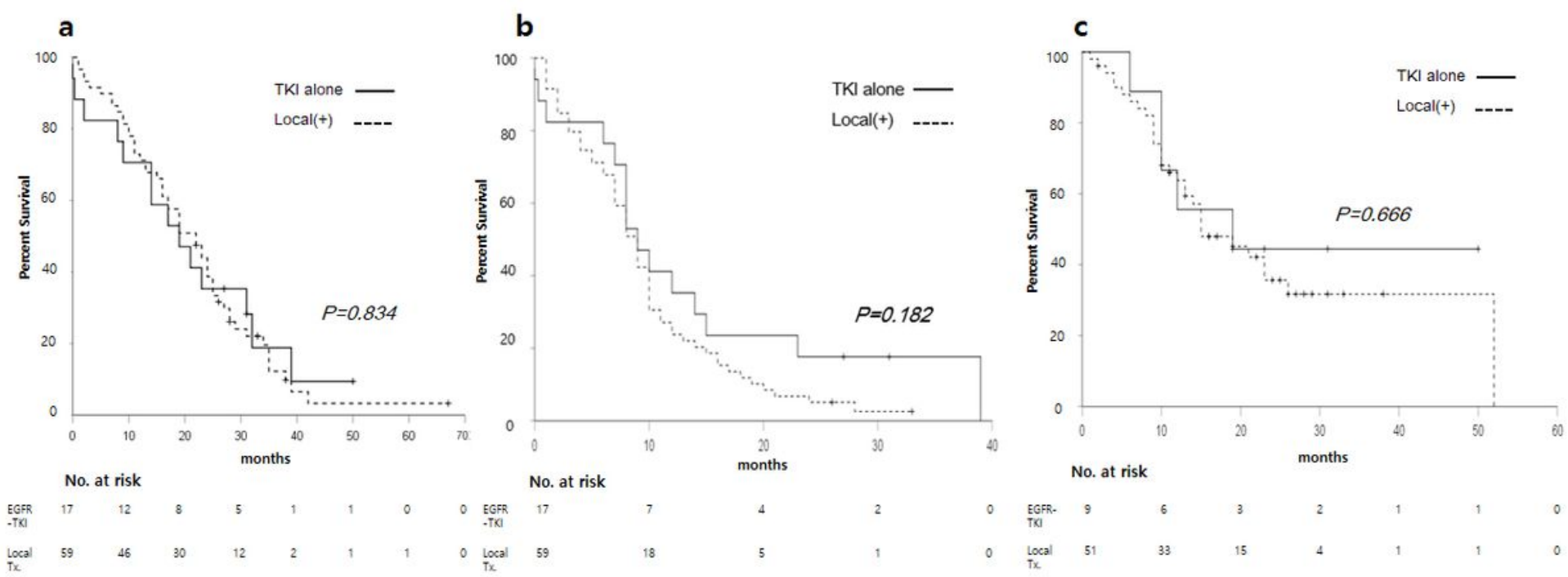

\section{Figure 2}

a Overall survival, b progression-free survival (PFS), and c intracranial-PFS of EGFR-mutant non-small cell lung cancer patients with brain metastasis according to treatment modality (EGFR-TKI vs. local treatment) 